\title{
Estudio piloto de la eficacia y de los efectos sobre los gametocitos del esquema artesunato-mefloquina-primaquina para la malaria por Plasmodium falciparum
}

\author{
Ana María Vásquez, Felipe Sanín, Luis Gonzalo Álvarez, Alberto Tobón, \\ Alexandra Ríos, Silvia Blair \\ Grupo de Malaria, Universidad de Antioquia, Medellín, Colombia.
}

Introducción. El tratamiento de la malaria por $P$. falciparum requiere de un esquema seguro, eficaz y de impacto en la transmisión. En 2006, se implementó en Antioquia el esquema artesunato-mefloquina y se adicionó primaquina para eliminar los gametocitos.

Objetivo. Evaluar la eficacia y acción gametocida de los esquemas artesunato-mefloquinaprimaquina y artesunato-mefloquina en pacientes con malaria no complicada por $P$. falciparum de Turbo, Antioquia.

Materiales y métodos. Ensayo clínico aleatorio; los tratamientos se suministraron de forma supervisada y se realizó seguimiento clínico-parasitológico en los días 1, 2, 3, 7, 14, 21, 28, 35, y 42, para evaluar la respuesta según el protocolo OMS-2003 modificado.

Resultados. Entre abril de 2007 y febrero de 2008, 50 pacientes fueron reclutados; los resultados mostraron una eficacia de 100\% (IC95\% 86,3\%-100\%) para el esquema artesunato-mefloquina (con/sin primaquina); la parasitemia y la fiebre fueron eliminadas completamente al tercer día de tratamiento en todos los pacientes. La eliminación de gametocitos fue mayor con el uso de primaquina; al tercer día de seguimiento, el 92\% (IC95\% 74\%-99\%) de los pacientes que recibieron primaquina no tuvieron gametocitos, en comparación con 78,3\% (IC95\% 59\%-93\%) de pacientes del grupo artesunato-mefloquina. Además, el esquema artesunato-mefloquinaprimaquina eliminó la gametocitemia una semana antes que el esquema sin primaquina.

Conclusión. Se recomienda el uso del esquema artesunato-mefloquina para la malaria por $P$. falciparum por su alta eficacia y se sugieren futuras evaluaciones del beneficio de la $P Q$ en la reducción de la densidad y prevalencia de gametocitos.

Palabras clave: Plasmodium falciparum, malaria, artesunato, mefloquina, primaquina, resultado del tratamiento.

Therapeutic efficacy of a regimen of artesunate-mefloquine-primaquine treatment for Plasmodium falciparum malaria and treatment effects on gametocytic development

Introduction. The treatment of Plasmodium falciparum malaria requires a safe and effective therapeutic treatment regimen, which in turn has high impact on the transmission. In 2006, an artesunate (AS)-mefloquine (MQ) treatment program was implemented in Antioquia. In addition, primaquine (PQ) was added to eliminate malaria gametocytes in the bloodstream.

Objective. The efficacy and gametocytocidal activity was evaluated for two treatment regimens, AS-MQ-PQ and AS-MQ, in patients with uncomplicated $P$. falciparum malaria.

Materials and methods. Between April 2007 and February 2008, 50 patients were recruited for the trial in Turbo, Antioquia. A randomized clinical trial was conducted. Treatment compliance was supervised, with a clinical and parasitological assessment on days 1, 2, 3, 7, 14, 21, 28, 35, and 42 to evaluate response rate according to the WHO 2003 protocol.

Results. Clinical response and parasite elimination efficacy of AS-MQ (with or without PQ) was $100 \%$ (95\% Cl $86.3 \%-100 \%)$, and parasitemia and fever were absent on day 3 of treatment in all patients. Gametocyte elimination was superior when PQ was used--92\% (95\% Cl: 74\%-99\%) of patients who received PQ had no gametocytes on day 3, compared to $78.3 \%$ (95\% Cl: $59 \%-93 \%$ ) of patients who only received AS-MQ. Furthermore, circulating gametocytes were eliminated on average one week faster when the AS-MQ-PQ treatment scheme was used compared to the scheme without $P Q$. 
Conclusion. These studies recommend the use of AS-MQ to treat $P$. falciparum malaria given its good therapeutic efficacy. However, further assessment is suggested concerning the benefit of adding $P Q$ to this treatment scheme.

Keywords: $P$. falciparum, malaria, artesunate, mefloquine, primaquine, treatment outcome.

La malaria es una infección parasitaria que constituye un grave problema de salud a nivel mundial y causa entre 300 y 500 millones de casos clínicos y entre 1,5 a 2,7 millones de muertes anuales, la mayoría, por Plasmodium falciparum. Al final de 2004, 107 países tenían áreas con riesgo de transmisión de la enfermedad, donde ha causado efectos devastadores en la salud, el desarrollo y el bienestar socioeconómico de las comunidades más pobres del mundo $(1,2)$.

Uno de los principales problemas para el control del paludismo, o malaria, por $P$. falciparum es la resistencia parasitaria a los antipalúdicos actualmente disponibles y las altas tasas de falla terapéutica a los esquemas de tratamiento (3). Posterior a los primeros informes de resistencia a cloroquina en los años cincuenta en Asia y en los años sesenta en Suramérica, la resistencia a este medicamento se ha reportado en todas las regiones del mundo donde hay transmisión de la enfermedad. Además, diversas cepas de $P$. falciparum han desarrollado resistencia a la mayoría de antipalúdicos de uso común, como la sulfadoxina-pirimetamina y mefloquina (4). La respuesta a los medicamentos antipalúdicos puede medirse mediante estudios in vivo 0 in vitro; los primeros miden la eficacia o respuesta clínica y parasitológica de los pacientes a los diferentes esquemas de tratamiento y permiten clasificarla como falla terapéutica o respuesta clínico-parasitológica adecuada (5), mientras que los segundos permiten determinar el fenotipo del parásito según su capacidad de sobrevivir o multiplicarse luego de ser sometido a medicamentos antipalúdicos en las concentraciones recomendadas (6).

\footnotetext{
Correspondencia:

Ana María Vásquez, Grupo de Malaria, Sede de Investigación Universitaria (SIU), Universidad de Antioquia, Calle 62 № 52-59, torre 1, piso 6, laboratorio 610, Medellín, Colombia Telefax: (574) 2196487 amvc.ana@gmail.com
}

Recibido: 16/04/08; aceptado:04/03/09
En Colombia, el panorama de resistencia y falla terapéutica se empieza a conocer desde 1961, cuando se reportó por primera vez falla a cloroquina. Las tasas de curación con este medicamento han disminuido drásticamente con reportes de falla hasta de $97 \%$ en el departamento de Antioquia (7) y hasta $70 \%$ en la costa Pacífica (8). En 2006, Blair y colaboradores reportaron cifras de falla a todos los medicamentos usados como monoterapia: a la cloroquina de $82 \%$, a la sulfadoxina-pirimetamina de $24,1 \%$, a la amodiaquina de $30,3 \%$, a la mefloquina de $4,4 \%$ y a las combinaciones cloroquina más sulfadoxinapirimetamina $(17,2 \%)$, artesunato más sulfadoxina-pirimetamina $(3,4 \%)$, amodiaquina más sulfadoxina-pirimetamina (2,2\%). Hasta este año, el tratamiento de primera línea para la malaria no complicada por $P$. falciparum en Colombia era la combinación amodiaquina más sulfadoxina-pirimetamina, con una eficacia del $98 \%$ y sin efectos secundarios graves (9).

Como medida para controlar la resistencia y las altas tasas de falla terapéutica, se ha recomendado la implementación de terapia antipalúdica combinada con derivados de la artemisinina, los medicamentos más eficaces, rápidos y potentes actualmente disponibles para el tratamiento de $P$. falciparum (10). Se entiende como terapia combinada el uso concomitante de dos o más fármacos esquizonticidas con mecanismos de acción independiente y blancos bioquímicos no relacionados, que mejoren la eficacia terapéutica y disminuyan el riesgo de desarrollar resistencia a los medicamentos individuales (11). Los estudios en Tailandia y África, y algunos en Suramérica, indican un enorme beneficio en la adición de artesunato a las monoterapias con amodiaquina, sulfadoxina-pirimetamina o mefloquina, al reducir significativamente el número de fallas terapéuticas, y se ha reportado un efecto gametocitocida en $P$. falciparum de los derivados de artemisinina, aspecto que contribuye al control de la transmisión (12-14). 
Actualmente, sólo se dispone de dos medicamentos con efecto en los gametocitos de $P$. falciparum: la primaquina y los derivados de artemisinina. La Organización Mundial de la Salud (OMS) sugiere que la adición de una dosis única de primaquina o de agentes gametocitocidas a las terapias antipalúdicas podría tener un efecto beneficioso en la reducción de la transmisión en zonas de baja transmisión; sin embargo, no hay pruebas al respecto (11). De igual forma, existe controversia sobre cuál de los dos tipos de medicamentos, los derivados de la artemisinina o de la primaquina, poseen mayor efecto sobre los gametocitos; sólo unos pocos estudios se han adelantado en zonas de alta transmisión y no han evaluado la combinación de ambos medicamentos (15). Se desconoce si el uso simultáneo de primaquina con derivados de la artemisinina dé como resultado una fuerte supresión en la gametocitemia y capacidad infecciosa de los gametocitos; esto es posible puesto que ambos medicamentos reducen la población de gametocitos y afectan su viabilidad (11).

El Ministerio de la Protección Social implementó en Antioquia en el 2006, nuevos esquemas de tratamiento para la malaria por $P$. falciparum con artesunato más mefloquina y adicionó primaquina para infertilizar los gametocitos (16). Se desconocen la eficacia y la seguridad de este nuevo esquema en la población colombiana, así como los efectos en la reducción de la prevalencia y la densidad de gametocitos cuando se suministra la primaquina con el artesunato.

El objetivo de este estudio fue evaluar la eficacia, la seguridad y la acción gametocida del esquema artesunato más mefloquina más primaquina frente a otro esquema igual pero sin primaquina, para definir la utilidad de ésta en pacientes con malaria no complicada por $P$. falciparum en Turbo, Antioquia.

\section{Materiales y métodos}

\section{Tipo de estudio}

Se trata de un ensayo clínico aleatorio en pacientes captados en el centro de salud Tablitas en Turbo, Antioquia, según el orden de llegada, con determinación no ciega del efecto en grupos iguales; se aplicó el protocolo de la OMS-2003 modificado por el Grupo Malaria (5,9). Cada paciente con malaria no complicada por $P$. falciparum se asignó a uno de los dos grupos de tratamiento: artesunato más mefloquina o artesunato más mefloquina más primaquina, mediante tabla de números aleatorios elaborada en el programa Epilnfo 6,04.

\section{Población y muestra}

El estudio se llevó a cabo en el municipio de Turbo, Antioquia, región de alta endemia palúdica, localizada en el Urabá antioqueño, con una población estimada para el año 2004 de 123.604 habitantes (49.187 en zona urbana y 74.417 en zona rural); en 2006 , se presentaron 5.674 casos de malaria (12,4\% por $P$. falciparum). Por ser un estudio piloto, el tamaño de la muestra fue por conveniencia.

\section{Criterios de inclusión}

Los criterios de inclusión fueron: 1) hombres o mujeres no embarazadas (según una prueba de tirilla reactiva) mayores de un año de edad con infección única por $P$. falciparum y parasitemia al ingreso entre 150 y 50.000 parásitos asexuados/ $\mu \mathrm{l}$; 2) ausencia de signos o síntomas de peligro o de complicación palúdica; 3) no presentar antecedentes de alergia a los antipalúdicos; 4) ausencia de enfermedad grave (diabetes, falla renal, cardiaca o respiratoria, cirrosis hepática, $\mathrm{VIH} /$ sida, enfermedad neuropsiquiátrica, desnutrición) al momento de diagnóstico de paludismo; 5) no haber recibido ningún tratamiento antipalúdico completo en las últimas 72 horas (o mefloquina en las cuatro últimas semanas), y 6) aceptar participar en el estudio, firmar el consentimiento informado y comprometerse a acudir a los siete controles durante el seguimiento.

\section{Diagnóstico de malaria y recuento de parásitos}

El diagnóstico se hizo mediante gota gruesa, la cual se tiñó con coloración de Field durante 10 minutos. Inicialmente, el diagnóstico lo hizo la microscopista del puesto de salud y las placas que resultaron positivas se entregaron a los investigadores para determinar la parasitemia y 
la gametocitemia; se realizó recuento de anillos y gametocitos contra un total de 200 leucocitos y el resultado se expresó como parásitos/ $\mu \mathrm{l}$ de sangre, usando como constante un total de 8.000 leucocitos por microlitro. La gota gruesa se consideró negativa si, después de observar 200 campos microscópicos (objetivo de 100X), no se encontraba ningún parásito.

\section{Evaluación clínica}

Cada uno de los pacientes fue evaluado el día de ingreso (día 0) por un médico y una bacterióloga con una experiencia de cinco años en estudios de eficacia, para determinar la presencia de síntomas o signos indicativos de infección palúdica y descartar la presencia de signos de peligro o de complicación. El tratamiento se suministró de manera supervisada para evaluar la tolerancia y facilitar su cumplimiento.

\section{Esquemas de tratamiento}

Cada esquema se administró en las dosis recomendadas por el Ministerio de la Protección Social de Colombia y la OMS-2006 (cuadro 1). A los pacientes del grupo de artesunato más mefloquina más primaquina, se les suministró una dosis de primaquina en forma única al final del tercer día.

\section{Seguimiento}

Se realizó control clínico y parasitológico a todos los pacientes, mediante búsqueda activa, los días 1, 2, 3, 7, 14, 21, 28, 35 y 42. Se registraron los efectos adversos, como aquellos signos o síntomas que aparecen durante el seguimiento y que no estaban presentes en el momento del ingreso, y aquéllos que, aun estando presentes, se intensificaron durante el seguimiento. Se consideró como efecto adverso serio aquél que comprometía la vida del paciente o involucraba su hospitalización. La relación entre los efectos adversos y el medicamento se clasificó como: ninguna, poco probable, posible, probable y definitiva (17).

\section{Análisis estadístico}

Los datos se analizaron en el programa estadístico SPSS, versión 10 (SPSS Inc. Chicago II) y en Excel (Microsoft Office 2007), y la base de datos se elaboró en Epilnfo 6.04. Se evaluó la normalidad de las variables densidad de parásitos asexuados y sexuados mediante la prueba de Shapiro-Wilks y no presentaron distribución normal, aun habiendo hecho transformación logarítmica y de otra índole. Por ello, los datos para estas dos variables se muestran en medianas con sus rangos intercuartílicos y los tratamientos se compararon por medio de la prueba no paramétrica de Mann-Whitney. La comparación de proporciones se realizó con la prueba de ji al cuadrado de Pearson y se consideró un nivel de significancia de $5 \%$.

\section{Consideraciones éticas}

El estudio fue avalado por el Comité Técnico de Investigaciones Médicas de la Facultad de Medicina de la Universidad de Antioquia, en su reunión del del 21 de junio del 2007, Acta 004.

\section{Resultados}

Durante el periodo comprendido entre abril de 2007 y febrero de 2008, se reclutaron 53 pacientes con diagnóstico de malaria por $P$. falciparum. Tres de los pacientes fueron excluidos por diagnóstico de enfermedad de base durante el seguimiento, malaria mixta o retiro voluntario.

\section{Características de la población}

Las características basales al momento de la admisión de los 25 pacientes asignados a cada grupo, fueron similares en ambos esquemas de tratamiento y se presentan en el cuadro 2. Los síntomas generales de cefalea y escalofrío estuvieron presentes en todos los pacientes en el momento de la admisión; también fueron comunes la sudoración (88\%), el dolor osteomuscular (84\%) y la astenia (95,3\%). Ninguno de los pacientes desarrolló enfermedad grave durante el seguimiento. Los principales hallazgos parasitológicos y de temperatura corporal en el día 0 se muestran en el cuadro 2.

\section{Evaluación de la eficacia terapéutica}

Durante los 42 días de seguimiento, no se presentó ninguna recurrencia clínica ni parasitológica con ninguno de los dos esquemas de 
tratamiento. Se obtuvo respuesta terapéutica adecuada en el 100\% (IC95\% 86,3\%-100\%) de los pacientes, tanto para el esquema artesunato más mefloquina más primaquina como para artesunato más mefloquina.

\section{Negativización de la parasitemia}

La proporción de pacientes con gota gruesa positiva para formas asexuadas de $P$. falciparum disminuyó rápidamente después de suministrar el tratamiento. En el primer día de seguimiento, más de la mitad de los pacientes resolvieron la infección con ambos esquemas; en el día 2, todos los pacientes del grupo de artesunato más mefloquina más primaquina no tuvieron parásitos asexuales en el examen microscópico, en comparación con $4 \%$ de pacientes positivos en el grupo de artesunato más mefloquina.

El promedio de parásitos asexuados/ $\mu$ lde sangre disminuyó rápidamente con la administración de ambos esquemas de tratamiento y sin diferencias estadísticamente significativas entre los dos esquemas, con una completa eliminación de la densidad parasitaria al tercer día de seguimiento.

\section{Resolución de la fiebre}

La desaparición de la fiebre fue más rápida en el grupo de artesunato más mefloquina. Durante el día 1 de evaluación, el $96 \%$ de los pacientes con este tratamiento estaban afebriles, en comparación con $84 \%$ del grupo de artesunato más mefloquina más primaquina. Un paciente del grupo de artesunato más mefloquina presentó temperatura axilar $\geq 37,5^{\circ} \mathrm{C}$ al día 7 de seguimiento, pero no tuvo ninguna asociación clínica ni parasitológica con el episodio anterior de malaria.

El comportamiento de la temperatura promedio durante los primeros siete días de seguimiento después de la administración de los esquemas de tratamiento, mostró una normalización de la misma a partir del primer día de seguimiento en ambos esquemas terapéuticos y la permanencia de rangos normales durante los días siguientes de evaluación.

\section{Efectos de los esquemas sobre los gametocitos}

Los gametocitos en el momento de la admisión estaban presentes en $20 \%$ de los pacientes,

Cuadro 1. Dosis de artesunato, mefloquina y primaquina suministradas según edad del paciente.

\begin{tabular}{|c|c|c|c|c|c|c|c|}
\hline \multirow{2}{*}{$\begin{array}{l}\text { Edad } \\
\text { años }\end{array}$} & \multicolumn{3}{|c|}{ AS (tabletas de 200 mg) } & \multicolumn{3}{|c|}{ MQ (tabletas de $250 \mathrm{mg}$ ) } & \multirow{2}{*}{$\begin{array}{c}P Q \text { (15 mg) (años) } \\
\text { Día } 2\end{array}$} \\
\hline & Día 0 & Día 1 & Día 2 & Día 0 & Día 1 & Día 2 & \\
\hline $1-6$ & 50 mg & $50 \mathrm{mg}$ & $50 \mathrm{mg}$ & & $250 \mathrm{mg}$ & & $0,3-0,6 \mathrm{mg} / \mathrm{kg}$ \\
\hline $7-13$ & $100 \mathrm{mg}$ & $100 \mathrm{mg}$ & $100 \mathrm{mg}$ & $250 \mathrm{mg}$ & $250 \mathrm{mg}$ & $250 \mathrm{mg}$ & $22,5 \mathrm{mg}$ \\
\hline$>13$ & $200 \mathrm{mg}$ & $200 \mathrm{mg}$ & $200 \mathrm{mg}$ & $500 \mathrm{mg}$ & $500 \mathrm{mg}$ & $500 \mathrm{mg}$ & $45 \mathrm{mg}$ \\
\hline
\end{tabular}

AS: artesunato; $M Q$ : mefloquina; $P Q$ : primaquina

Cuadro 2. Características de base de los pacientes con malaria por P. falciparum involucrados en el estudio.

\begin{tabular}{lcc}
\hline & AS-MQ-PQ $(\mathbf{n}=\mathbf{2 5})$ & AS-MQ $(\mathbf{n}=\mathbf{2 5})$ \\
\cline { 2 - 3 } & Percentil 50 (25-75) & Percentil 50 (25-75) \\
\hline Edad & $23,8(12,4-37,1)$ & $20(13,6-41,1)$ \\
Parasitemia & $3.120(940-5.440)$ & $3.280(1.220-7.140)$ \\
Gametocitemia & $0(0-0)$ & $0(0-20)$ \\
Temperatura axilar (으) & $(36,4-38,4)$ & $37,4(36,7-38,2)$ \\
Antecedentes de malaria/vida & $0(0-1)$ & $0(0-2)$ \\
Uso previo CQ $\mathrm{n}(\%)$ & $2(8)$ & $4(16)$ \\
Pacientes febriles $\mathrm{n}(\%)$ & $11(44)$ & $10(40)$ \\
Pacientes con gametocitemia n (\%) & $4(16)$ & $6(24)$ \\
\hline
\end{tabular}

AS: artesunato; $\mathrm{MQ}$ : mefloquina; $\mathrm{PQ}$ : primaquina $\mathrm{CQ}$ : cloroquina 
lo cual indica cronicidad de la infección de 7 a 10 días. La eliminación de gametocitos fue más eficaz con el esquema de tratamiento de artesunato más mefloquina más primaquina; al segundo día de seguimiento, 92\% (IC95\% $74 \%-99 \%$ ) de los pacientes de este grupo no tenían formas sexuadas en la gota gruesa, en comparación con 78,3\% (IC 95\%: 59\%-93\%) de los del grupo deartesunato más mefloquina (figura 1). La densidad promedio de gametocitos/ $\mu$ l de sangre se ilustra en la figura 2. La persistencia de gametocitos circulantes al día 14 de seguimiento no presentó diferencias estadísticamente significativas entre ambos esquemas y la eliminación fue completa en el día 14 con artesunato más mefloquina más primaquina $y$, en el día 21, con artesunato más mefloquina.

\section{Seguridad de los esquemas de tratamiento}

Ambos esquemas de tratamiento fueron bien tolerados por todos los pacientes y no se reportaron efectos adversos serios. Los efectos adversos más comunes fueron el vértigo en $42 \%$ de los pacientes y alteraciones de tipo motor como marcha inestable en una proporción de $36 \%$; éstos aparecieron entre los días 2 y 3 de seguimiento. También, fueron frecuentes alteraciones de tipo gastrointestinal, como diarrea, epigastralgia, náuseas, retortijones y vómito. Los eventos adversos más frecuentes por tratamiento se ilustran en la figura 3.

Se estableció una relación definitiva entre el tratamiento antipalúdico y el vértigo en $67 \%$ de los pacientes $\mathrm{y}$, con marcha inestable, en $61 \%$; además, se encontró un alto porcentaje de relación probable entre el medicamento y las alteraciones gastrointestinales (figura 4).

\section{Discusión}

Debido a la extensión de la resistencia de $P$. falciparum a los antipalúdicos suministrados como monoterapia y a la resistencia cruzada observada entre medicamentos estructuralmente relacionados o con mecanismos de acción similares, la terapia antipalúdica combinada ha surgido en los últimos años como el tratamiento de primera línea para la malaria no complicada por $P$. falciparum (11).
Los primeros estudios de Tailandia, región con alta prevalencia de cepas de $P$. falciparum multirresistentes, evidenciaron que la adición de artesunato a la monoterapia con mefloquina y el aumento en la dosis de esta última, proporcionaban altas tasas de curación de la infección (entre 90\% y 100\%) acompañadas de una reducción en la resistencia a la mefloquina y en la incidencia clínica de la malaria (13). Hallazgos similares se observaron en diferentes regiones de África (18). Desde entonces, varios países adoptaron la combinación como tratamiento de primera línea para $P$. falciparum en Asia, África y Suramérica (11).

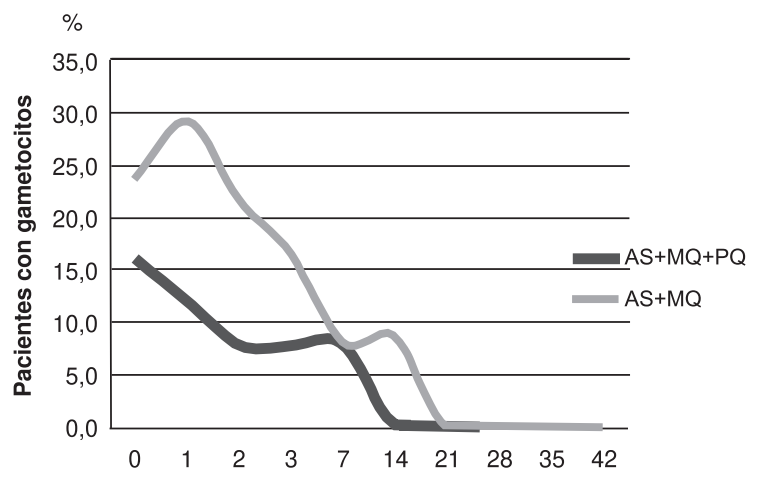

Figura 1. Porcentaje de pacientes con gota gruesa positiva para gametocitos de $P$. falciparum durante el seguimiento, según el esquema de tratamiento recibido.

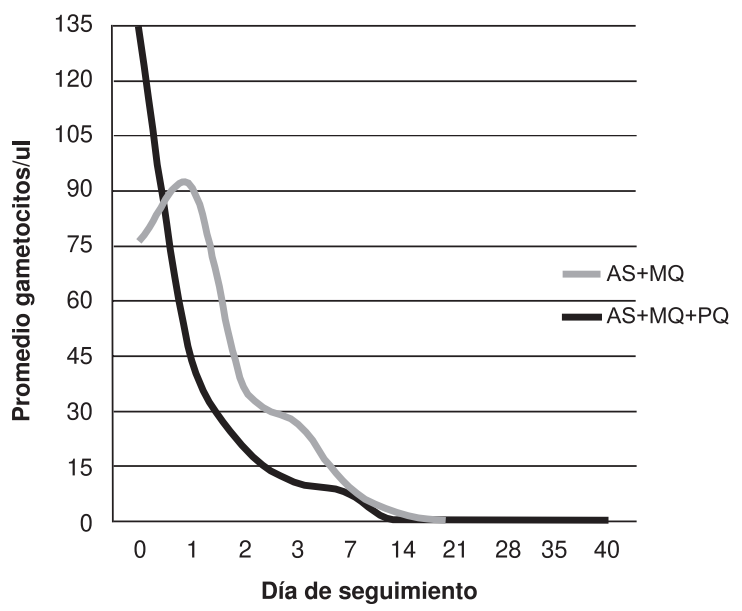

Figura 2. Comportamiento de la densidad de gametocitos/ul de sangre de $P$. falciparum después de suministrar cada uno de los esquemas de tratamiento. 


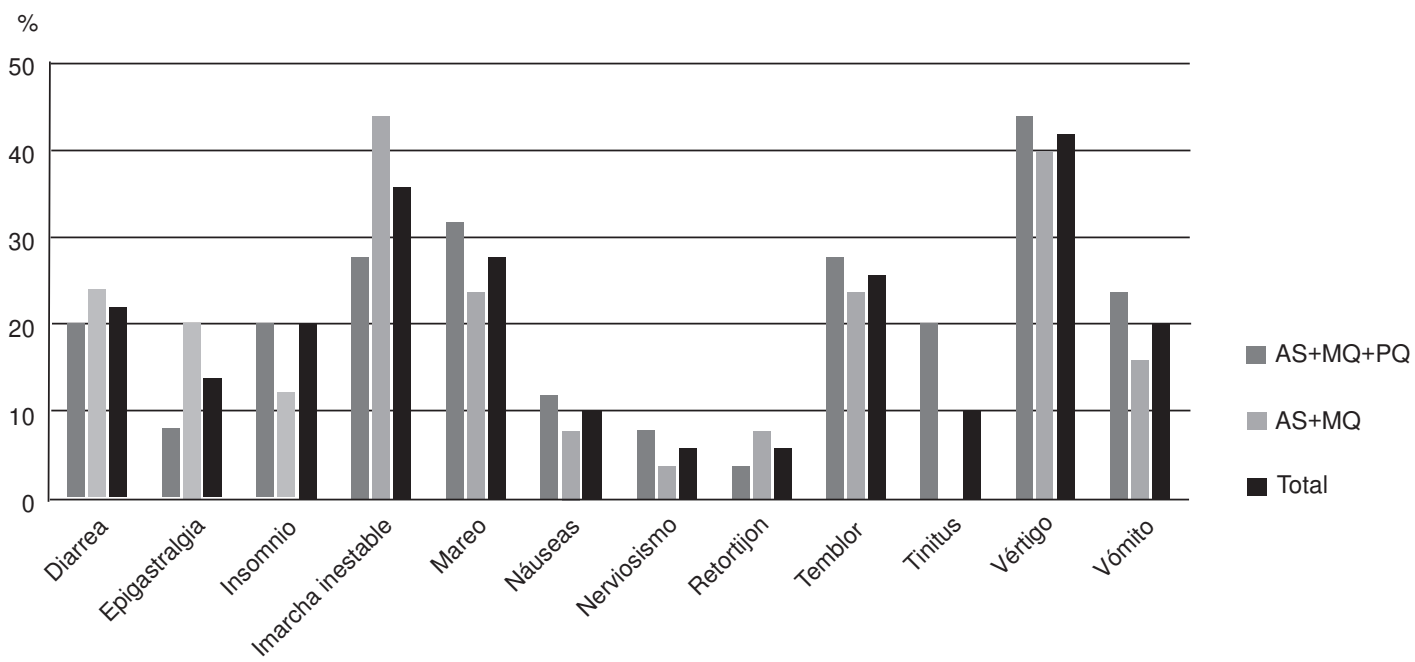

Figura 3 . Proporción de eventos adversos más frecuentes por tratamiento en los pacientes.

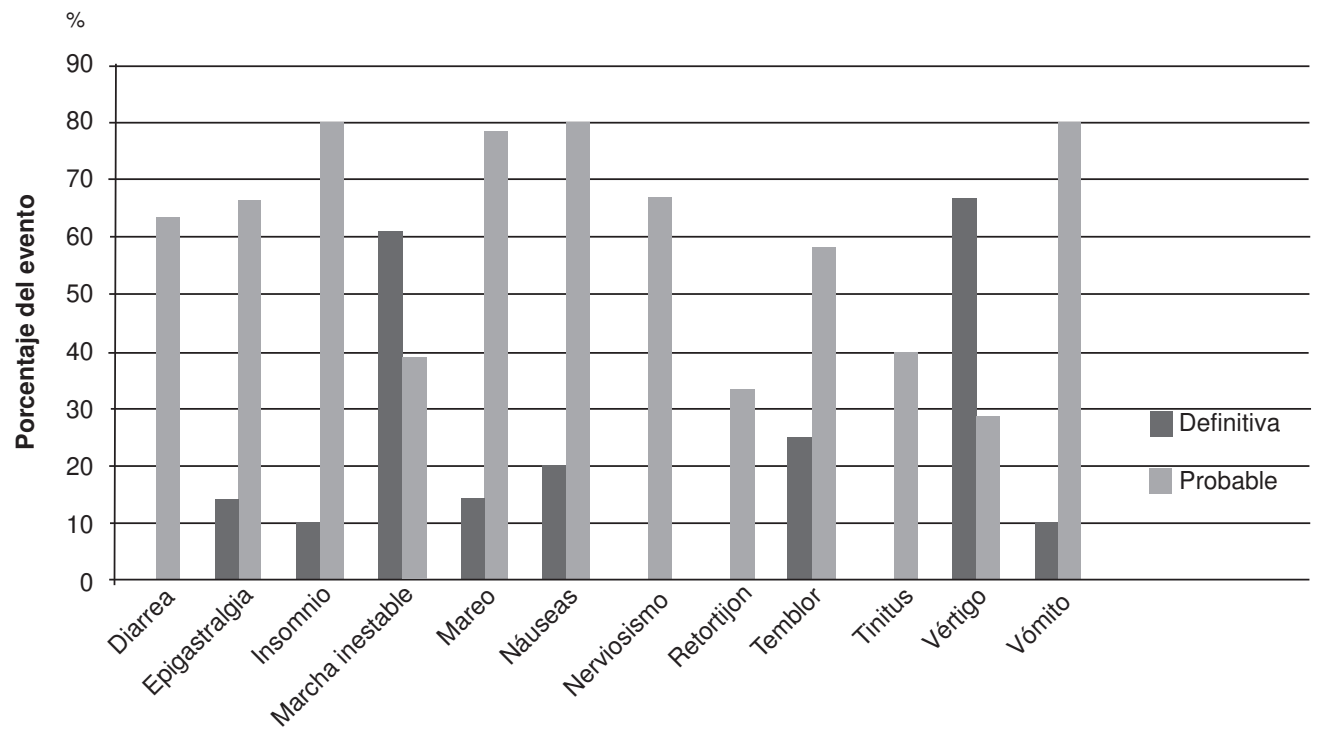

Figura 4. Proporción de los eventos adversos con una relación definitiva o probable con el medicamento antipalúdico.

El presente estudio evaluó por vez primera la eficacia en Colombia del esquema de tratamiento artesunato más mefloquina con primaquina y sin ella para la malaria no complicada por $P$. falciparum. Los resultados demuestran que el esquema de tratamiento es muy eficaz, la tasa de curación es del 100\% y la desaparición de los síntomas y la parasitemia ocurren rápidamente. Al tercer día de suministrado el tratamiento no había pacientes febriles ni con parasitemia. Hubo hallazgos similares en la mayoría de países que han adoptado esta combinación. La eficacia de esta combinación en diferentes regiones del mundo se resume en el cuadro 3.

Los resultados concuerdan con lo reportado previamente en nuestro continente, en países como Perú y Bolivia. El primer país en adoptar el esquema de tratamiento de artesunato más mefloquina fue Perú en el 2001. Los estudios iniciales sobre la eficacia terapéutica de la 
combinación arrojaron excelentes resultados, con una buena respuesta terapéutica en el $100 \%$ de los casos (28). El segundo país en adoptar este esquema como tratamiento de primera línea fue Bolivia y los resultados de las tasas de curación de pacientes también fueron del 100\% (32), al igual que los estudios preliminares en Venezuela (XII Congreso de la Asociación Panamericana de Infectología, VI Congreso Venezolano de Infectología, II Simposio Latinoamericano y del Caribe de Infecciones de Transmisión Sexual, 15 al 18 de mayo de 2005. Caracas, Venezuela. http://caibco.ucv.ve). En estos estudios, los síntomas y la parasitemia asexual se eliminaron rápidamente después de suministrar el tratamiento (aproximadamente, en el tercer día de seguimiento) y también se observó un acentuado beneficio de la combinación de artesunato más mefloquina en la reducción de la gametocitemia. Los estudios reportados por RAVREDA, que evaluaron la combinación de artesunato más mefloquina en Suramérica, muestran resultados similares: entre 2002 y 2005 se reportó un estudio en Guyana, donde se encontró una proporción en la falla total de $1,2 \%$, mientras que en Surinam se informaron dos estudios con $2 \%$ de falla (42).

La combinación de artesunato más mefloquina ha sido bien tolerada por los pacientes en todos los países donde se ha implementado, con presencia de efectos adversos menores. En el presente estudio no se observaron efectos adversos serios con ninguno de los dos esquemas; los más comunes fueron el vértigo y las alteraciones motoras, como marcha inestable, que se reportan frecuentemente en la terapia

Cuadro 3. Tasas de curación de la combinación artesunato más mefloquina según reportes de diferentes localizaciones geográficas hasta 2007 y con diferentes dosis de los medicamentos.

\begin{tabular}{|c|c|c|c|c|c|c|}
\hline \multirow[t]{2}{*}{ Lugar } & \multirow[t]{2}{*}{ Año } & \multicolumn{2}{|c|}{ Dosis (mg/kg) } & \multirow[t]{2}{*}{ Curación (\%) } & \multirow[t]{2}{*}{ IC 95\% } & \multirow[t]{2}{*}{ Referencia } \\
\hline & & AS & $M Q$ & & & \\
\hline Tailandia & 1994 & 10 & 25 & 98 & $96,5-99,5$ & 19 \\
\hline Myanmar & 1995 & 12 & 25 & 86,1 & $81-91$ & 20 \\
\hline Tailandia & 1996 & 4 & 20 & 97 & $86-99$ & 21 \\
\hline Tailandia & 1997 & 4 & 20 & 100 & $92,7-100$ & 22 \\
\hline Tailandia & 1997 & 12 & 20 & 98,7 & $90,3-100$ & 23 \\
\hline Tailandia & 1997 & 12 & 25 & 89 & $87,6-90,4$ & 24 \\
\hline Tailandia & 1998 & 12 & 25 & 94 & $87,4-100$ & 25 \\
\hline Tailandia & 1998 & 12 & 25 & 95,7 & $93,4-98$ & 26 \\
\hline Camerún & 2002 & 12 & 15 & 100 & $93-100$ & 27 \\
\hline Perú & 2003 & 12 & 15 & 100 & $93-100$ & 28 \\
\hline Myanmar & 2004 & 12 & 15 & 79 & $69,7-88,3$ & 29 \\
\hline Laos & 2004 & 12 & 25 & 100 & $93-100$ & 30 \\
\hline \multirow{3}{*}{ Myanmar } & 2004 & 4 & 15 & 94,1 & $90,7-97,5$ & 31 \\
\hline & & 4 & 25 & 96,4 & $93,8-99$ & \\
\hline & & 12 & 25 & 100 & $98-100$ & \\
\hline Bolivia & 2004 & 12 & 15 & 100 & $94,8-100$ & 32 \\
\hline Bangladesh & 2005 & 12 & 25 & 100 & $96,5-100$ & 33 \\
\hline Tailandia & 2005 & 12 & 25 & 96,3 & $93,9-98,7$ & 34 \\
\hline Venezuela & 2005 & 12 & 25 & 100 & $94-100$ & \\
\hline Tailandia & 2005 & 12 & 25 & 100 & $90,2-100$ & 35 \\
\hline India & 2006 & 12 & 15 & 93,6 & $88,8-98,4$ & 36 \\
\hline \multirow[t]{2}{*}{ Tailandia } & 2006 & 12 & 15 & 94,2 & $84-98,8$ & 37 \\
\hline & & 12 & 25 & 92 & $88-96$ & \\
\hline Myanmar & 2006 & 12 & 25 & 100 & $97,7-100$ & 38 \\
\hline Cambodia & 2006 & 12 & 25 & 94,5 & $91,5-97,5$ & 39 \\
\hline Senegal & 2007 & 12 & 15 & 100 & $97,5-100$ & 40 \\
\hline Kenia & 2006 & & & 98,4 & $96,2-100$ & 41 \\
\hline
\end{tabular}

$M Q$ : mefloquina; $A S$ : artesunato 
con mefloquina y que se presentaron en igual proporción en los dos esquemas evaluados (14).

Las alteraciones gastrointestinales también fueron frecuentes en ambos grupos de tratamiento; los más comunes fueron diarrea, epigastralgia, náuseas, retortijones y vómito. Estos eventos son muy comunes durante el tratamiento con derivados de artemisinina pero, también, son de común presentación durante el tratamiento con mefloquina $(14,43)$.

Llama la atención la presencia de acúfenos en el $12 \%$ de los pacientes incluidos en el estudio, todos tratados con artesunato más mefloquina más primaquina, lo que indica que puede tener relación con la presencia de la primaquina en este esquema de tratamiento. Comúnmente, se le atribuye a la quinina y sus derivados efectos ototóxicos que producen hipoacusia neurosensorial acompañada de síntomas como zumbidos, vértigo, náuseas y vómito, y alteraciones de la visión. La quinina y sus derivados causan ototoxicidad cualquiera que sea la vía de administración, debido a una degeneración de las células ciliadas delórgano de Corti $(44,45)$. En un estudio dirigido a evaluar los efectos ototóxicos del tratamiento antipalúdico en 725 pacientes, en Mozambique, se encontró que $78 \%$ de los pacientes tratados con antipalúdicos desarrolló hipoacusia neurosensorial, y la quinina y la cloroquina fueron los dos fármacos con mayor capacidad de lesionar el vestíbulo (46). No existe ningún reporte que relacione la primaquina con otoxicidad, pero tal efecto puede ser posible, ya que estos tres medicamentos pertenecen al grupo de las quinolinas.

La primaquina (8-aminoquinolina) es un antipalúdico ampliamente utilizado como gametocitocida contra los estadios sexuales de $P$. falciparum. Los estudios in vivo en voluntarios han demostrado que este medicamento acelera la negatividad de gametocitos circulantes y reduce potencialmente su capacidad infecciosa (47). En áreas de baja transmisión, se recomienda el uso de una dosis única de primaquina durante el último día de tratamiento del esquema adoptado. Entre los antipalúdicos actualmente disponibles, sólo la primaquina y los derivados de la artemisinina tienen efecto en la reducción y viabilidad de los gametocitos (11).

El efecto de los tratamientos de artesunato más mefloquina más primaquina y artesunato más mefloquina sobre los gametocitos, fue evaluado en términos de porcentaje de pacientes con gametocitemia y promedio de la densidad de gametocitos/ $\mu$ l de sangre después de suministrar los tratamientos completos. En ambas variables se encontraron diferencias entre los dos esquemas: se observó una menor proporción de pacientes con gametocitos circulantes después del suministro de artesunato más mefloquina más primaquina, esta proporción de pacientes positivos fue de $0 \%$ en el día 14 de seguimiento, mientras la ausencia total de pacientes con gametocitos en el esquema artesunato más mefloquina se presentó en el día 21 de seguimiento. La negatividad de la densidad promedio de gametocitos/ $\mu$ l en sangre presenta un comportamiento similar al observado en la proporción de pacientes con gametocitemia, al observarse persistencia de gametocitos circulantes en el día 14 de seguimiento en el grupo con artesunato más mefloquina, en comparación con el esquema de artesunato más mefloquina más primaquina, que no presentó gametocitemia detectable por microscopia en el día 14, aunque dichas diferencias no fueron estadísticamente significativas $(p>0,05)$.

Los estudios in vitro han demostrado la superioridad del artesunato sobre la primaquina para prevenir la transmisión de la malaria, debido a que ejerce un potente efecto sobre la capacidad infecciosa de los gametocitos en Anopheles dirus: a una concentración de 1,5 ng/ $\mathrm{ml}$, inhibe el $90 \%$ de dicha capacidad; además, en cultivos de gametocitos con 24 horas de exposición al artesunato, se han observado cambios morfológicos en los gametocitos que no son evidentes con la exposición a la primaquina (47). Sin embargo, como el artesunato y su metabolito dehidroartemisinina son eliminados muy rápidamente del sistema sanguíneo (11), los ensayos in vitro representan un modelo de exposición prolongada al medicamento que puede no ocurrir in vivo, por lo cual se 
puede estar sobreestimando el efecto sobre la transmisión de este antipalúdico.

Los efectos in vivo en humanos del uso de la artemisinina y sus derivados en la transmisión de la malaria, muestran una reducción significativa de gametocitos circulantes (48). Con la introducción del esquema de artesunato más mefloquina en Tailandia, se ha observado una disminución de la resistencia a la mefloquina y una reducción en la incidencia clínica de malaria que, posiblemente, obedezcanaladisminución de gametocitos como resultado del medicamento (13). Sin embargo, un estudio reciente, realizado en septiembre del 2006 en el este de Tanzania, demostró que al día 14 de seguimiento el 62,7\% de los pacientes que recibieron el esquema de artesunato más sulfadoxina-pirimetamina permanecían con gametocitemias submicroscópicas detectadas mediante PCR. Dichas gametocitemias se eliminaron de forma eficiente con una dosis única de primaquina adicional al artesunato más sulfadoxina-pirimetamina; por lo tanto, se concluye que la primaquina es muy eficiente produciendo negatividad de las gametocitemias microscópicas y submicroscópicas que permanecen después del tratamiento con artesunato más sulfadoxina-pirimetamina y se demuestra el significativo valor aditivo de la primaquina en el esquema de primera línea para el tratamiento para $P$. falciparum en la reducción de la gametocitemia y de la transmisibilidad (49).

En un estudio que comparó la eficacia del artesunato más mefloquina y de la primaquina más mefloquina en la eliminación de gametocitos, no se encontraron diferencias significativas entre ambos esquemas de tratamiento, ya que las gametocitemias persistentes o el desarrollo de gametocitemias subsiguientes al suministro del tratamiento, fueron similares en ambos grupos, por lo cual los autores sugieren dirigir las estrategias de control de la transmisión hacia la rápida eliminación de las formas asexuadas de $P$. falciparum para prevenir la subsiguiente gametocitemia. En este sentido, los derivados de la artemisinina son una excelente opción (15).

El mecanismo de acción por medio del cual los compuestos derivados de la artemisinina disminuyen la gametocitemia circulante, obedece principalmente a una rápida eliminación de las formas asexuadas y de los gametocitos inmaduros de $P$. falciparum que constituyen la fuente para los gametocitos maduros $(15,48)$. Por otra parte, la primaquina ejerce su mecanismo de acción mediante la eliminación de gametocitos maduros y de ahí surge la importancia de incluirla en los esquemas de tratamiento para disminuir la transmisión. Sin embargo, son pocos los estudios que evalúan la eficacia de las combinaciones terapéuticas con derivados de la artemisinina más primaquina, para producir negatividad de la gametocitemia circulante y para disminuir la capacidad infecciosa de los gametocitos, después de suministrado este tratamiento. Es de esperarse que el uso de ambos mejore las medidas de control sobre la transmisión de la malaria $(11,50)$. Esta mejoría en la negatividad de la gametocitemia adicionando primaquina al esquema de artesunato más mefloquina pudo evidenciarse en el presente estudio, pues se logró la reducción de la gametocitemia microscópica, al menos con una semana de anterioridad en comparación con el tratamiento de artesunato más mefloquina; no obstante, dichos resultados deben confirmarse en un estudio clínico con un mayor número de pacientes.

Los resultados de este estudio piloto demostraron una tasa de curación del $100 \%$ en los pacientes con $P$. falciparum que recibieron el esquema de artesunato más mefloquina y que, actualmente, constituye la primera línea de tratamiento en Antioquia. Además, indican un aparente beneficio en el uso de una dosis única de primaquina para contribuir al control de la transmisión de la malaria, por lo que se sugiere evaluar en futuros estudios la utilidad de adicionar primaquina al esquema de tratamiento para $P$. falciparum en las zonas colombianas endémicas para malaria.

\section{Agradecimientos}

A la Dirección Seccional de Salud de Antioquia, en especial, a Armando Galeano, a la Universidad de Antioquia, al personal de salud del puesto de diagnóstico de malaria "Tablitas", especialmente, a Rosángela Tamayo y Fernando Méndez, por 
su constante colaboración y a los pacientes que participaron en el estudio.

\section{Conflictos de intereses}

No existe ningún conflicto de intereses.

\section{Financiación}

Este proyecto fue realizado en el marco del contrato RC-398-2006, registrado con el código 1115-343-19157 de Colciencias, cofinanciado por la Dirección Seccional de Salud de Antioquia y la Universidad de Antioquia.

\section{Referencias}

1. WHO-UNICEF. Roll-back malaria. World Malaria Report 2005. Geneva: World Health Organization; 2005. Fecha de consulta: diciembre de 2007. Disponible en: http:// www.rbm.who.int/wmr2005.

2. Phillips RS. Current status of malaria and potential for control. Clin Microbiol Rev. 2000;14:208-26.

3. Bloland PB. Drug resistance in malaria. WHO/CDS/CSR/ DRS/2001.4. Geneva: World Health Organization; 2001.

4. Wongsrichanalai C, Pickard AL, Wernsdorfer WH, Meshnick SR. Epidemiology of drug-resistant malaria. Lancet Infect Dis. 2002;2:209-18.

5. WHO. Assessment and monitoring of antimalarial drug efficacy for the treatment of uncomplicated falciparum malaria. WHO/HTM/RBM/2003.50. Geneva: World Health Organization; 2003.

6. WHO. Monitoring antimalarial drug resistance. WHO/ CDS/CSR/EPH/2002.17. Geneva: World Health Organization; 2001.

7. Blair S, López M, Piñeros J, Álvarez T, Tobón A, Carmona J. Eficacia terapéutica de tres esquemas de tratamiento de malaria no complicada por Plasmodium falciparum, Antioquia, Colombia, 2002. Biomédica. 2003;23:318-27.

8. Osorio LE, Giraldo LE, Grajales LF, Arriaga AL, Andrade AL, Ruebushll T, et al. Assessment of therapeutic response of Plasmodium falciparum to chloroquine and sulfadoxine/pyrimethamine in a low malaria transmission area in Colombia. Am J Trop Med Hyg. 1999;61:968-72.

9. Blair S, Lacharme L, Carmona-Fonseca J, Piñeros J, Ríos A, Álvarez T, et al. Therapeutic efficacy test in malaria falciparum in Antioquia, Colombia. Malar J. 2006;5:14.

10. Orjuela P, González I, Osorio L. Terapia combinada como estrategia en la prevención de la resistencia a los antimaláricos. Biomédica. 2004;24:423-37.

11. WHO. Guidelines for the treatment of malaria. WHO/HTM/ MAL/2006.1108. Geneva: World Health Organization; 2006.
12. WHO. Susceptibility of Plasmodium falciparum to antimalarial drugs monitoring antimalarial drug resistance. Report on global monitoring 1996-2004. WHO/HTM/MAL/ 2005.1103. Geneva: World Health Organization; 2005.

13. Nosten F, van Vugt M, Price R, Luxemburger C, Thway $\mathrm{KL}$, Brockman A, et al. Effects of artesunate-mefloquine combination on incidence of Plasmodium falciparum malaria and mefloquine resistance in western Thailand: a prospective study. Lancet. 2000;356:297-302.

14. Davis TM, Karunajeewa HA, llett KF. Artemisinin-based combination therapies for uncomplicated malaria. Med $\mathrm{J}$ Aust. 2005;182:181-5.

15. Suputtamongkol Y, Chindarat S, Silpasakorn S, Chaikachonpatd S, Lim K, Chanthapakajee K, et al. The efficacy of combined mefloquine-artesunate versus mefloquine-primaquine on subsequent development of Plasmodium falciparum gametocitemia. Am J Trop Med Hyg. 2003;68:620-3.

16. Dirección Seccional de Salud de Antioquia. Protocolo de vigilancia epidemiológica para malaria, Antioquia 2007. Fecha de consulta: diciembre de 2007. Disponible en: http://www.dssa.gov.co/dowload/P0841.pdf

17. Talisuna A, Staedke S, D'Alessandro U. Pharmacovigilance of antimalarial treatment in Africa: is it possible? Malar J. 2006;5:50.

18. Davis T. Addition of artesunate to standard antimalarial drugs reduces treatment failure. Evidence Based Healthcare. 2004;8:156-8.

19. Nosten F, Luxemburger C, ter Kuile FO, Woodrow C, Eh JP, Chongsuphajaisiddhi T, et al. Treatment of multidrug-resistant Plasmodium falciparum malaria with 3-day artesunate-mefloquine combination. J Infect Dis. 1994;170:971-7.

20. Price RN, Nosten F, Luxemburger C, Kham A, Brockman A, Chongsuphajaisiddhi T, et al. Artesunate versus artemether in combination with mefloquine for the treatment of multidrug-resistant falciparum malaria. Trans R Soc Trop Med Hyg. 1995;89:523-7.

21. Bunnag D, Kanda T, Karbwang J, Thimasarn K, Pungpak S, Harinasuta T. Artemether or artesunate followed by mefloquine as a possible treatment for multidrug resistant falciparum malaria. Trans $\mathrm{R}$ Soc Trop Med Hyg. 1996;90:415-7.

22. Bunnag D, Kanda T, Karbwang J, Thimasarn K, Pungpak S, Harinasuta T. Two doses of artemether/ mefloquine or artesunate/mefloquine combination for multidrug resistant falciparum malaria. Southeast Asian J Trop Med Public Health. 1997;28:727-30.

23. Thimasarn K, Sirichaisinthop J, Chanyakhun P, Palananth C, Rooney W. A comparative study of artesunate and artemether in combination with mefloquine on multidrug resistant falciparum malaria in eastern Thailand. Southeast Asian J Trop Med Public Health. 1997;28:465-71. 
24. Price RN, Nosten F, Luxemburger C, van Vugt M, Phaipun L, Chongsuphajaisiddhi T, et al. Artesunate/ mefloquine treatment of multi-drug resistant falciparum malaria. Trans R Soc Trop Med Hyg. 1997;91:574-7.

25. Price R, Luxemburger C, van Vugt M, Nosten F, Kham A, Simpson J, et al. Artesunate and mefloquine in the treatment of uncomplicated multidrug-resistant hyperparasitaemic falciparum malaria. Trans R Soc Trop Med Hyg. 1998;92:207-11.

26. van Vugt M, Brockman A, Gemperli B, Luxemburger C, Gathmann I, Royce C, et al. Randomized comparison of artemether-benflumetol and artesunate-mefloquine in treatment of multidrug-resistant falciparum malaria. Antimicrob Agents Chemother. 1998;42:135-9.

27. Massougbodji A, Kone M, Kinde-Gazard D, SameEkobo A, Cambon N, Mueller EA. A randomized, double-blind study on the efficacy and safety of a practical three-day regimen with artesunate and mefloquine for the treatment of uncomplicated Plasmodium falciparum malaria in Africa. Trans $\mathrm{R}$ Soc Trop Med Hyg. 2002;96:655-9.

28. Marquiño W, Huilca M, Calampa C, Falconí E, Cabezas C, Naupay R, et al. Efficacy of mefloquine and a mefloquine-artesunate combination therapy for the treatment of uncomplicated Plasmodium falciparum malaria in the Amazon Basin of Peru. Am J Trop Med Hyg. 2003;68:608-12.

29. Smithuis F, Shahmanesh M, Kyaw MK, Savran O, Lwin S, White NJ. Comparison of chloroquine, sulfadoxine/ pyrimethamine, mefloquine and mefloquine-artesunate for the treatment of falciparum malaria in Kachin State, North Myanmar. Trop Med Int Health. 2004;9:1184-90.

30. Stohrer JM, Dittrich S, Thongpaseuth V, Vanisaveth V, Phetsouvanh R, Phompida S, et al. Therapeutic efficacy of artemether-lumefantrine and artesunatemefloquine for treatment of uncomplicated Plasmodium falciparum malaria in Luang Namtha Province, Lao People's Democratic Republic. Trop Med Int Health. 2004;9:1175-83.

31. Smithuis F, van der Broek I, Katterman N, Kyaw MK, Brockman A, Lwin S, et al. Optimising operational use of artesunate-mefloquine: a randomized comparison of four treatment regimens. Trans R Soc Trop Med Hyg. 2004;98:182-92.

32. Avila JC, Villaroel R, Marquiño W, Zegarra J, Mollinedo R, Ruebush TK. Efficacy of mefloquine and mefloquine-artesunate for the treatment of uncomplicated Plasmodium falciparum malaria in the Amazon region of Bolivia. Trop Med Int Health. 2004;9:217-21.

33. van den Broek IV, Maung UA, Peters A, Liem L, Kamal M, Rahman M, et al. Efficacy of chloroquine+sulfadoxinepyrimethamine, mefloquine+artesunate and artemether +lumefantrine combination therapies to treat Plasmodium falciparum malaria in the Chittagong Hill Tracts, Bangladesh. Trans R Soc Trop Med Hyg. 2005;99:727-35
34. Hutagalung R, Paiphun L, Ashley EA, McGready R, Brockman A, Thwai $K L$, et al. A randomized trial of artemether-lumefantrine versus mefloquine-artesunate for the treatment of uncomplicated multi-drug resistant Plasmodium falciparum on the western border of Thailand. Malar J. 2005;4:46.

35. Tangpukdee N, Krudsood S, Thanachartwet W, Chalermrut K, Pengruksa C, Srivilairit S, et al. An open randomized clinical trial of Artekin vs. artesunatemefloquine in the treatment of acute uncomplicated falciparum malaria. Southeast Asian J Trop Med Public Health. 2005;36:1085-91.

36. Campbell P, Baruah S, Narain K, Rogers C. A randomized trial comparing the efficacy of four treatment regimens for uncomplicated falciparum malaria in Assam state, India. Trans R Soc Trop Med Hyg. 2006; 100: 108-18.

37. Vijaykadga S, Rojanawatsirivej C, Cholpol S, Phoungmanee D, Nakavej A, Wongsrichanalai C. In vivo sensitivity monitoring of mefloquine monotherapy and artesunate-mefloquine combinations for the treatment of uncomplicated falciparum malaria in Thailand in 2003. Trop Med Int Health. 2006;11:211-9.

38. Smithuis F, Kyaw MK, Phe O, Aye KZ, Htet L, Barends $\mathbf{M}$, et al. Efficacy and effectiveness of dihydroartemisininpiperaquine versus artesunate-mefloquine in falciparum malaria: an open-label randomized comparison. Lancet. 2006;367:2075-85

39. Denis MB, Tsuyuoka R, Poravuth Y, Narann TS, Seila S, Lim C, et al. Surveillance of the efficacy of artesunate and mefloquine combination for the treatment of uncomplicated falciparum malaria in Cambodia. Trop Med Int Health. 2006;11:1360-6.

40. Faye B, Ndiaye JL, Ndiaye D, Dieng Y, Faye O, Gaye O. Efficacy and tolerability of four antimalarial combinations in the treatment of uncomplicated Plasmodium falciparum malaria in Senegal. Malar J. 2007;6:80.

41. Bhatt KM, Samia BM, Bhatt SM, Wasunna KM. Efficacy and safety of an artesunate/mefloquine combination (artequin) in the treatment of uncomplicated $P$. falciparum malaria in Kenya. East Afr Med J. 2006;83:236-42.

42. RAVREDA-AMI. Antimalarial drug efficacy studies 2002-2005. Fecha de consulta: septiembre de 2008. Disponible en: http://www.paho.org/spanish/AD/DPC/ $\mathrm{CD} /$ ravreda-ami-areas.htm

43. Giraldo C, Blair S. Mefloquina: revisión de tema IATREIA. 2003;16:19-31.

44. Berninger E, Karlsson K, Alvan G. Quinine reduces the dynamic range of the human auditory system. Acta Otolaryngol. 1998;118:46-51.

45. Tange RA, Dreschler WA, Claessen FA, Perenboom RM. Ototoxic reactions of quinine in healthy persons and patients with Plasmodium falciparum infection. Auris Nasus Larynx. 1997;24:131-6. 
46. Simoes Da Silva MV. Estudio de los efectos ototóxicos en 725 pacientes tratados con antimaláricos en el Hospital Central de Maputo (Mozambique) (tesis). Barcelona: Universidad Autónoma de Barcelona; 2004.

47. Chotivanich K, Sattabongkot J, Udomsangpetch R, Looareesuwan S, Day NP, Coleman RE, et al. Transmission-blocking activities of quinine, primaquine, and artesunate. Antimicrob Agents Chemother. 2006;50:1927-30.

48. Davis ME, Karunajeewa HA, llet KF. Artemisinin-based combination therapies for uncomplicated malaria. Med $\mathrm{J}$ Aust. 2005;182:181-5.
49. Shekalaghe S, Drakeley C, Gosling R, Ndaro A, van Meegeren M, Enevold A, et al. Primaquine clears submicroscopic Plasmodium falciparumgametocytes that persist after treatment with sulphadoxine-pyrimethamine and artesunate. PloS ONE. 2007;2:e1023. Doi:10.1371/ journal.-pone.0001023

50.Pukrittayakamee S, Chotivanich K, Chantra A, Clemens R, Looareesuwan S, White NJ. Activities of artesunate and primaquine against asexual- and sexualstage parasites in falciparum malaria. Antimicrob Agents Chemother. 2004;48:1329-34. 